\title{
Severe hypermagnesemia presenting with abnormal electrocardiographic findings similar to those of hyperkalemia in a child undergoing peritoneal dialysis
}

\author{
Won Kyoung Jhang, MD, PhD, Yoon Jung Lee, MD, Young A Kim, MD, Seong Jong Park, MD, PhD, Young Seo Park, MD, PhD \\ Department of Pediatrics, Asan Medical Center Children's Hospital, University of Ulsan College of Medicine, Seoul, Korea
}

In this report, we present a pediatric case of severe symptomatic hypermagnesemia resulting from the use of magnesium oxide as a laxative in a child undergoing continuous cyclic peritoneal dialysis for end-stage renal disease. The patient showed abnormal electrocardiography (ECG) findings, such as tall T waves, a widened QRS complex, and irregular conduction, which were initially misdiagnosed as hyperkalemia; later, the correct diagnosis of hypermagnesemia was obtained. Emergent hemodialysis successfully returned the serum magnesium concentration to normal without complications. When abnormal ECG changes are detected in patients with renal failure, hypermagnesemia should be considered.

Key words: Magnesium, Peritoneal dialysis, Hyperkalemia
Corresponding author: Young Seo Park, MD, PhD Department of Pediatrics, Asan Medical Center Children's Hospital, University of Ulsan College of Medicine, 88 Olympic-ro 43-gil, Songpa-gu, Seoul 138-736, Korea

Tel: +82-2-3010-3376, Fax: +82-2-473-3725

E-mail: yspark@amc.seoul.kr

Received: 3 December, 2011

Revised: 17 April, 2012

Accepted: 12 July, 2012

\section{Introduction}

Hypermagnesemia is an uncommon clinical condition. Symptomatic hypermagnesemia usually occurs in elderly individuals and in patients with bowel disorders or renal insuffciency in the setting of excessive magnesium ingestion ${ }^{1-5)}$. In people with normal renal function, the kidneys have a very large capacity for magnesium excretion. However, in dialysis patients, the dialysis plays a main role in magnesium removal, such that serum and dialysate magnesium concentrations are related, with both being normal or slightly elevated, depending on the dialysis solution used for these patients ${ }^{6}$.

The potential for misdiagnosis of hypermagnesemia in these patients may be high, not only because magnesium concentration is not measured routinely but because the initial symptoms of hypermagnesemia are nonspecific ${ }^{7)}$. In addition, the electrocardiographic manifestations of hypermagnesemia are very similar to those of hyperkalemia, a more general complication observed in patients with renal insufficiency.

We describe here a pediatric patient undergoing continuous cyclic peritoneal dialysis (CCPD) for renal failure who developed symptomatic hypermagnesemia but was originally misdiagnosed with hyperkalemia.

\section{Case report}

An 11-year-old boy on CCPD was admitted to the emergency room (ER) after 1 week of lethargy, decreased oral intake and poor function of CCPD. He had a medical history

Copyright (C 2013 by The Korean Pediatric Society

This is an open-access article distributed under the terms of the Creative Commons Attribution NonCommercial License (http://creativecommons.org/ licenses/by-nc/3.0/) which permits unrestricted noncommercial use, distribution, and reproduction in any medium, provided the original work is properly cited. 
of mild cerebral palsy and steroid resistant focal segmental glomerulosclerosis leading to end stage renal disease. He had been managed with CCPD for 5 months. His medications included 4 types of antihypertensive drugs (nifedipine, enalapril, losartan, and amlodipine), 3 types of antiepileptic drugs (oxcarbazepine, clobazam, and lamotrigine), iron, calcium, and vitamin supplementation for chronic renal failure. Additionally, as a laxative for chronic constipation, lactulose had been administered for 5 months. Since lactulose didn't improve his symptom much, magnesium oxide was administered for 2 weeks prior to admission.

At admission, his blood pressure was $112 / 75 \mathrm{mmHg}$, his heart rate was $135 \mathrm{bpm}$, and his respiratory rate was 32 breaths/ min. Physical examination revealed no specific abnormalities. Laboratory tests showed a leukocyte count of $7,900 / \mathrm{mm}^{3}$, a hemoglobin concentration of $10.7 \mathrm{~g} / \mathrm{dL}$, a serum sodium concentration of $132 \mathrm{mmol} / \mathrm{L}$, a serum potassium concentration of 7.2 $\mathrm{mmol} / \mathrm{L}$, a blood urea nitrogen concentration of $53 \mathrm{mg} / \mathrm{dL}$, a serum creatinine concentration of $9.3 \mathrm{mg} / \mathrm{dL}$ and a C-reactive protein concentration of $1.1 \mathrm{mg} / \mathrm{dL}$. His dialysate fluid was clear. While in the ER, his temperature rose to $38.4^{\circ} \mathrm{C}$ and his lethargy persisted. He was diagnosed with a viral infection and hyperkalemia and was admitted for general supportive care. After admission, an electrocardiography (ECG) revealed a tall T wave, a wide QRS complex and irregular conduction. CCPD was much frequently performed every 1 hour and he was administered intravenous calcium gluconate and regular insulin with glucose to correct his supposed hyperkalemia. Follow-up laboratory tests from blood drawn 4 hours later showed that his potassium concentration had decreased to $6.3 \mathrm{mmol} / \mathrm{L}$, but his ECG findings did not improve and even became aggravated to wide QRS complex with irregular conduction abnormalities (Fig. 1A). At that time, we found that his serum concentration of magnesium was markedly elevated, to $8.9 \mathrm{mg} / \mathrm{dL}(7.4 \mathrm{mEq} / \mathrm{L})$, and he was diagnosed with symptomatic severe hypermagnesemia.

The patient was transferred to the pediatric intensive care unit and emergently hemodialyzed for hyperkalemia and hypermagnesemia. The patient underwent hemodialysis for three hours with dialysate containing $2.0 \mathrm{mEq} / \mathrm{L}(2.4 \mathrm{mg} / \mathrm{dL})$ of magnesium, without heparin treatment. His serum concentration of magnesium decreased to $5.7 \mathrm{mg} / \mathrm{dL}(4.7 \mathrm{mEq} / \mathrm{L})$ after 30 minutes. An ECG performed during the dialysis revealed a normal sinus rhythm with mild T wave changes (Fig. 1B). The patient tolerated hemodialysis well and showed no hemodynamic instability. After 3 hours of hemodialysis, his serum concentrations of magnesium and potassium had decreased to $3.7 \mathrm{mg} / \mathrm{dL}$ and $4.8 \mathrm{mmol} / \mathrm{L}$, respectively. On the following day, he was transferred to a general ward and continued CCPD every 2 hours. After two days, however, his serum magnesium concentration rose slightly, to $4.7 \mathrm{mg} / \mathrm{dL}$. A repeat session of hemodialysis reduced his serum magnesium concentration to $3.17 \mathrm{mg} / \mathrm{dL}$, which was thereafter maintained within a normal range with CCPD. At the time of discharge, his serum electrolytes and ECG findings were within the normal range.

\section{Discussion}

Magnesium, the fourth most abundant cation and the second most common intracellular electrolyte, serves various important physiological functions ${ }^{6,8)}$, including facilitating the activities of more than 300 enzyme systems and being essential for the production and proper function of DNA ${ }^{7)}$. One of the primary functions of magnesium is to balance the activities of calcium in intracellular fluids that affect many organ systems such as the central nervous and cardiovascular systems, and neuromuscular junctions ${ }^{1)}$.

In the cardiovascular system, magnesium regulates the sodiumpotassium-adenosine triphosphatase pump, thus affecting the intracellular/extracellular potassium ratio and the resting membrane potential of myocardial cells. Magnesium also inhibits the influx of calcium through sarcolemmal channels and modulates

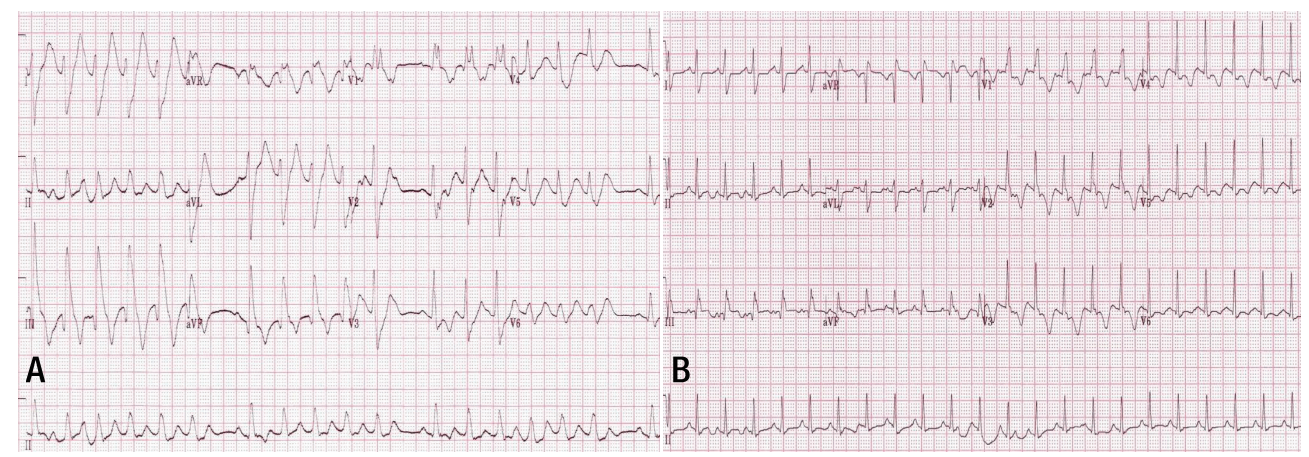

Fig. 1. Patient's electrocardiography (ECG) showing a tall T wave, a widened QRS complex, and irregular conduction with escape beats (A). Follow-up ECG after 3 hours of emergent hemodialysis, showing a normal sinus rhythm with a narrow QRS complex and a mild T wave change (B). 
cyclic adenosine monophosphate, thus blocking the slow inward calcium current. The cardiovascular system is, therefore, influenced by these calcium channel blocking properties and the electrophysiological effects of magnesium are similar to those of potassium $^{2)}$.

The presentation of hypermagnesemia depends on serum magnesium concentrations. Signs and symptoms of mild hypermagnesemia are usually subtle and nonspecific and may include flushing, warmth, nausea, headache, and lightheadedness. However, magnesium concentrations of 6-12 mg/dL (5-10 mEq/L) result in characteristic ECG changes, including prolongation of the PR interval, increased duration of QRS complex, prolonged QT interval, delayed intraventricular condiction, and increased height of the T wave, changes similar to those of hyperkalemia. Magnesium concentrations of 9-12 mg/dL may induce somnolence, loss of deep tendon reflex, and hypotension, and concentrations $>12 \mathrm{mg} / \mathrm{dL}$ (10 mEq/L) may result in sinoatrial and atrioventricular block, ventricular arrhythmias, muscle paralysis, hypoventilation, and stupor. Serum magnesium concentrations exceeding 15.6 $\mathrm{mg} / \mathrm{dL}$ (13 mEq/L) may result in cardiac asystole, coma and respiratory arrest ${ }^{3,8-10)}$.

Our patient, who had poor function of CCPD for a couple of days and used magnesium-containing laxatives for chronic constipation, developed hypermagnesemia, which presented with sinus arrest, wide QRS complex and ventricular arrhythmia. Due to his underlying cerebral palsy, other minor hypermagnesemic symptoms and signs could not be detected earlier, with symptoms becoming apparent only after serum magnesium concentrations reached a certain level. Moreover, this patient was initially diagnosed with and treated for hyperkalemia because the latter occurs more frequently in these patients.

There have been several reports where iatrogenic hypermagnesemia was induced by magnesium-containing drugs with or without renal dysfuncion ${ }^{1,3,4)}$. Magnesium containing laxatives should not be used in patients with renal failure.

Constipation is very common problem in dialysis patients, which causes drainage problems on peritoneal dialysis and it is therefore important to avoid becoming constipated. The general approach is to encourage daily activity. Then a laxative is given. Despite many different types of laxatives, a clear guideline for the patients with renal failure has been scarce. Thus, osmotic agent such as lactulose or stool softener such as docusate sodium could be recommended with caution with a consideration of drug compliance, drug interaction or side effects ${ }^{11-14)}$.

Treatment of hypermagnesemia consists of discontinuing the source of magnesium, respiratory and hemodynamic support, calcium supplementation, and treatment with diuretics and hemodialysis ${ }^{8,9)}$. Calcium not only reverses the effects of hypermagnesemia, such as respiratory depression, cardiac arrhythmias, hypotension and neuromuscular blockade by its antagonistic action but also helps increase renal excretion of magnesium.

Hemodialysis may be necessary for patients with renal dysfunction who experience hypermagnesemia or even for patients with normal renal function who have a massive overdose of magnesium-containing compounds ${ }^{3)}$. Indications for hemodialysis include renal failure, increasing magnesium levels despite diuresis, arrhythmias, and persistent hemodynamic instability.

This patient also required emergent hemodialysis due to poor function of CCPD and arrhythmias. Hemodialysis in this patient rapidly decreased both the serum magnesium and potassium concentrations.

In summary, severe symptomatic hypermagnesemia may result from magnesium containing medications, which are generally considered benign. It should not be used in patients with renal failure even with CCPD. Our findings indicate that the ECG manifestations of hypermagnesemia are very similar to those of hyperkalemia, increasing the potential for misdiagnosis of hypermagnesemia. Serum magnesium concentrations should be measured in patients with renal insufficiency who present with these symptoms, thus preventing significant complications and possible fatal events.

\section{Conflict of interest}

No potential conflict of interest relevant to this article was reported.

\section{References}

1. Birrer RB, Shallash AJ, Totten V. Hypermagnesemia-induced fatality following epsom salt gargles(1). J Emerg Med 2002;22:185-8.

2. Harker HE, Majcher TA. Hypermagnesemia in a pediatric patient. Anesth Analg 2000;91:1160-2.

3. Onishi S, Yoshino S. Cathartic-induced fatal hypermagnesemia in the elderly. Intern Med 2006;45:207-10.

4. Kontani M, Hara A, Ohta S, Ikeda T. Hypermagnesemia induced by massive cathartic ingestion in an elderly woman without preexisting renal dysfunction. Intern Med 2005;44:448-52.

5. Vissers RJ, Purssell R. Iatrogenic magnesium overdose: two case reports. J Emerg Med 1996;14:187-91.

6. Musso CG. Magnesium metabolism in health and disease. Int Urol Nephrol 2009;41:357-62.

7. Ali A, Walentik C, Mantych GJ, Sadiq HF, Keenan WJ, Noguchi A. Iatrogenic acute hypermagnesemia after total parenteral nutrition infusion mimicking septic shock syndrome: two case reports. Pediatrics 2003;112(1 Pt 1):e70-2.

8. Topf JM, Murray PT. Hypomagnesemia and hypermagnesemia. Rev Endocr Metab Disord 2003;4:195-206.

9. McGuire JK, Kulkarni MS, Baden HP. Fatal hypermagnesemia in a child treated with megavitamin/megamineral therapy. Pediatrics 2000;105:E18.

10. Swaminathan R. Magnesium metabolism and its disorders. Clin 
Biochem Rev 2003;24:47-66.

11. Lembo A, Camilleri M. Chronic constipation. N Engl J Med 2003; 349:1360-8.

12. Singh S, Rao SS. Pharmacologic management of chronic constipation. Gastroenterol Clin North Am 2010;39:509-27.
13. Xing JH, Soffer EE. Adverse effects of laxatives. Dis Colon Rectum 2001;44:1201-9.

14. Frazao JM, Cobum JW. Safety of magnesium laxatives in dialysis patients. Semin Dial 2007;10:292. 